\title{
Temperatura da pele de diferentes áreas corporais de idosos sem risco para lesões por pressão
}

Skin temperature of different body areas of the elderly without risk for pressure ulcer

Temperatura de la piel de diferentes áreas corporales de los ancianos sin riesgo de lesiones por presión

\author{
Rhea Silvia Soares ${ }^{\mathrm{I}}$, Suzinara Beatriz Soares de Lima ${ }^{\mathrm{II}}$, Thaís Dresch Eberhardt ${ }^{\mathrm{III}}$, Lidiana \\ Batista Teixeira Dutra Silveira ${ }^{\mathrm{IV}}$, Bruna Rossarola Pozzebonv ${ }^{\mathrm{v}}$ Liane Rocha Rodrigues ${ }^{\mathrm{VI}}$, \\ Paulo Jorge Pereira Alves ${ }^{\mathrm{VII}}$
}

\begin{abstract}
Resumo: Objetivo: identificar a temperatura da pele de diferentes áreas corporais de idosos hospitalizados em unidade de clínica cirúrgica sem risco de desenvolver lesões por pressão a partir da Escala de Braden. Método: estudo correlacional descritivo, com corte transversal, realizado em unidade de clínica cirúrgica de um hospital universitário de maio a outubro de 2017, com 84 pacientes. Realizada análise estatística descritiva. Resultados: a região sacral apresentou média de temperatura mais alta e o calcâneo direito a mais baixa. Não há diferença na temperatura da pele entre os lados direito e esquerdo nas escápulas, trocânteres e calcâneo; entre os sexos e raças. Quando mensurada das 9:00 às 13:00h, a temperatura dos calcâneos foi menor do que quando mensurada das 13:01 às 17:00h na região dos calcâneos. Conclusão: a região sacral apresenta a média mais alta de temperatura da pele, em relação às áreas mensuradas. Há simetria entre os lados corporais.
\end{abstract}

Descritores: Enfermagem geriátrica; Pele; Temperatura cutânea; Lesão por pressão; Idoso

\footnotetext{
${ }^{I}$ Doutora em Enfermagem pelo Programa de Pós-Graduação em Enfermagem da Universidade Federal de Santa Maria (PPGEnf UFSM), Professora do Colégio Politécnico da UFSM, Santa Maria, RS, Brasil. E-mail: rheasilviasoares@yahoo.com.br. Orcid: 0000-0001-87262377

II Doutora em enfermagem pela Escola de Enfermagem Anna Nery - Universidade Federal do Rio de Janeiro. Professora Associada do Departamento de Enfermagem da UFSM. Chefe da Divisão de Enfermagem do Hospital Universitário de Santa Maria, Santa Maria, RS, Brasil. E-mail: suzibslima@yahoo.com.br. Orcid: 0000-0002-2162-8601
}

III Doutora em Enfermagem pelo Programa de Pós-Graduação em Enfermagem da Universidade Federal de Santa Maria (PPGEnf UFSM), Professora da Universidade de Passo Fundo, Passo Fundo, RS, Brasil. E-mail: thaiseberhardt@gmail.com. Orcid: 0000-0003-0138-2066

IV Mestre em Enfermagem pelo PPGEnf UFSM, Doutoranda em Enfermagem pelo PPGEnf UFSM, Santa Maria, RS, Brasil. E-mail: lidianadutrasilveira@gmail.com. Orcid: 0000-0001-6373-9353

V Acadêmica do Curso de Graduação em Enfermagem da Universidade Federal de Santa Maria (UFSM). Bolsista Programa de Bolsas de Iniciação Científica da Fundação de Amparo à Pesquisa do Estado do Rio Grande do Sul, Santa Maria, RS, Brasil. E-mail: brunarpozzebon@gmail.com. Orcid: 0000-0002-5734-5355

VI Enfermeira pela Universidade Federal de Santa Maria, São Sepé, RS, Brasil. E-mail: lianerocharodrigues@yahoo.com.br. Orcid: 0000-0002-2903-7718

VII Doutor em Enfermagem pela Universidade Católica Portuguesa, Professor Auxiliar convidado no Instituto de Ciências da Saúde Porto da Universidade Católica de Portugal, Porto, Portugal. E-mail: pjpalves@gmail.com. Orcid: 0000-0002-6348-3316 
Temperatura da pele de diferentes áreas corporais de idosos sem risco para lesões por ... | 2

\begin{abstract}
Objective: to identify the skin temperature of different body areas of elderly inpatients at a surgical clinic unit without risk of developing pressure injuries from the Braden Scale. Method: descriptive correlational study, with cross-sectional design, conducted at a surgical clinic unit of a university hospital from May to October 2017, with 84 patients. Descriptive statistical analysis was performed. Results: the sacral region presented the highest mean temperature and the right calcaneus, the lowest. There is no difference in skin temperature between the right and left sides in the scapulae, trochanters and calcaneus; between the sexes and races. When measured from 9:00 a.m. to 1:00 p.m., the temperature of the calcaneus was lower than when measured from 1:01 p.m. to 5:00 p.m. in the calcaneus region. Conclusion: the sacral region presents the highest mean skin temperature in relation to the areas measured. There is symmetry between the body sides.
\end{abstract}

Descriptors: Geriatric nursing; Skin; Skin temperature; Pressure ulcer; Aged

Resumen: Objetivo: identificar la temperatura de la piel de diferentes áreas corporales de ancianos hospitalizados en una unidad clínica quirúrgica sin riesgo de desarrollar lesiones por presión a partir de la Escala Braden. Método: estudio correlativo descriptivo, con sección transversal, realizado en una unidad clínica quirúrgica de un hospital universitario de mayo a octubre de 2017, con 84 pacientes. Se realizó un análisis estadístico descriptivo. Resultados: la región sacra presentó una temperatura media más alta y el calcáneo derecho tenía la más baja. No hay diferencia en la temperatura de la piel entre los lados derecho e izquierdo en las escápulas, trocánteres y calcáneos; entre los sexos y las razas. Cuando se mide de 9:00 a.m. a 1:00 p.m., la temperatura del calcáneo fue menor que cuando se midió de 1:01 p.m. a 5:00 p.m. en la región del calcáneo. Conclusión: la región sacra presenta la temperatura media más alta de la piel, en relación con las áreas medidas. Hay simetría entre los lados del cuerpo.

Descriptores: Enfermería geriátrica; Piel; Temperatura cutánea; Úlcera por presión; Anciano

\title{
Introdução
}

O corpo humano possui vários processos de proteção contra diversos agentes agressores. A pele, como maior órgão do corpo humano, tem função protetora importante e eficiente na regulação da temperatura corporal e protege em relação a alguns fatores físicos como umidade e forças mecânicas - fricção e cisalhamento. As células da pele são renovadas constantemente de acordo com a exposição e região do corpo. ${ }^{1}$ Com o avanço da idade, a pele torna-se mais frágil e suas estruturas ficam menos resistentes aos processos agressores. A pele torna-se mais suscetível às ações do tempo, apresentando riscos ao desenvolvimento de lesão por pressão: pele fina e seca, com presença de edema, úmida e pegajosa e descorada. ${ }^{2}$

A temperatura da pele é apontada na literatura como um importante fator de risco indireto para o desenvolvimento das lesões por pressão (LPs), pois afeta a tolerância do tecido à 
3 | Soares RS, Lima SBS, Eberhardt TD, Silveira LBTD, Pozzebon BR, Rodrigues LR, et al.

pressão e ao cisalhamento. ${ }^{3-9}$ LP pode ser definida como um dano localizado na pele e/ou tecidos moles subjacentes, geralmente sobre uma proeminência óssea ou relacionada ao uso de dispositivo médico ou a outro artefato, ${ }^{10}$ podendo ser considerada como um problema de saúde pública, ${ }^{11}$ uma vez que apresenta elevadas taxas de incidência em unidades de internação $(24,3 \%)^{12}$ e em unidades de tratamento intensivo $(20,6 \%) .{ }^{13}$

A avaliação de risco é um componente importante da prática clínica dos profissionais, que visa identificar indivíduos com características que aumentam a probabilidade de desenvolvimento de LP, o que permite a implementação de um plano de cuidados para minimizar os fatores de risco. ${ }^{14}$ Assim, destaca-se a escala de Braden como uma das escalas mais utilizadas no mundo. Foi proposta por Braden e Bergstrom, em 1987, a partir de um esquema conceitual que envolve os fatores de risco e causais das LPs, também foi traduzida e validada no Brasil por Paranhos e Santos. ${ }^{15}$

Nesse sentido, estudos têm apontado que manter uma temperatura menor nas áreas de proeminência óssea parece ser um fator protetor para o aparecimento dessas lesões. ${ }^{4-5,9,16}$ Entretanto, ainda não se sabe quais seriam os valores considerados "normais" para esta variável. ${ }^{9}$ Nesse sentido, avaliar pacientes idosos, sem risco de desenvolver LP, pode fornecer valores de temperatura de sítios cutâneos que venham a contribuir, juntamente com outros estudos, como parâmetros clínicos, uma vez que ainda não é possível afirmar quais são os valores considerados normais. O que justifica a realização desta pesquisa é a necessidade de produzir conhecimento sobre a temática com diferentes populações e cenários para que, no futuro, seja possível estabelecer valores de referência, que poderão nortear ações na prática clínica dos enfermeiros.

A temperatura e a umidade da pele (microclima) do paciente são alteradas sempre que este estiver posicionado em uma cama ou cadeira, em contato com roupas, curativos ou dispositivos médicos. A pele se adapta continuamente a essas diferentes condições, assim, extremos de alta e baixa temperatura e umidade afetam negativamente a barreira da pele, 
Temperatura da pele de diferentes áreas corporais de idosos sem risco para lesões por ... $\mid 4$

contribuindo para um maior risco de LP. Os parâmetros específicos destas variáveis e possíveis limiares superior e inferior são atualmente desconhecidos, mas espera-se que seja específico da população e da área da pele. ${ }^{9}$

Diante desse contexto, tem-se como questão de pesquisa: em idosos hospitalizados em unidade de clínica cirúrgica sem risco de desenvolver lesões por pressão a partir da Escala de Braden, qual a temperatura da pele em diferentes áreas corporais? O estudo teve como objetivo identificar a temperatura da pele de diferentes áreas corporais de idosos hospitalizados em unidade de clínica cirúrgica sem risco de desenvolver lesões por pressão a partir da Escala de Braden.

\section{Método}

Trata-se de um estudo correlacional descritivo, ${ }^{17}$ com corte transversal. O projeto matricial teve como finalidade identificar a temperatura da pele de diferentes áreas corporais de pacientes internados em unidade de clínica cirúrgica, sem risco de desenvolver lesão por pressão. Para análise da presente pesquisa, foram incluídos somente os pacientes idosos (a partir de 60 anos de idade). ${ }^{18}$

A pesquisa foi realizada em uma unidade de clínica cirúrgica de um hospital universitário do sul do Brasil. A unidade possui 52 leitos e atende pacientes de alta complexidade no pré e pós-operatório das especialidades de cirurgia geral, traumatologia, cabeça e pescoço, torácica, proctologia, urologia e vascular. Participaram do projeto matricial pacientes hospitalizados na unidade de clínica cirúrgica que atenderam aos seguintes critérios de inclusão:

a) pacientes sem risco de desenvolver lesões por pressão a partir da Escala de Braden (19 a 23 pontos);

b) idade maior ou igual a 18 anos.

E como critérios de exclusão:

a) presença de lesão por pressão nas áreas de mensuração das variáveis; 
5 | Soares RS, Lima SBS, Eberhardt TD, Silveira LBTD, Pozzebon BR, Rodrigues LR, et al.

b) amputação de algum membro; presença de curativo, tala gessada ou fixador que impedisse o acesso aos locais de mensuração das variáveis;

c) nível de consciência alterado ou dificuldade de comunicação e sem acompanhante;

d) com presença de edema nos locais de mensuração das variáveis.

Para a realização do cálculo amostral do projeto matricial, foi utilizado o programa Epi Info $^{\mathrm{TM}}$, para estudos descritivos, considerado um poder estatístico de $80 \%$, um nível de significância de 95\% $(\alpha<0,05)$, tamanho populacional de 574 (número estimado de pacientes internados na unidade de clínica cirúrgica em um ano e sem risco de desenvolver LP), frequência esperada de alteração na temperatura da pele de $50 \%$ e margem de erro de 5 pontos percentuais. Assim, o resultado do cálculo amostral foi de 230 pacientes. Nesta análise, foram incluídos todos os idosos do banco de dados do projeto matricial, totalizando 84 pacientes, por meio de autorização do pesquisador responsável.

A equipe de coleta de dados foi composta por três enfermeiras, alunas da pós-graduação em enfermagem e três alunos de iniciação científica, que eram acadêmicos de enfermagem. Previamente ao início da coleta de dados, todos os coletadores receberam treinamento teóricoprático, com base no manual do coletador, o qual foi elaborado pelos pesquisadores para servir como guia para a coleta dos dados da pesquisa.

A coleta de dados teve início em maio de 2017, com entrada sucessiva dos indivíduos que atenderam aos critérios de seleção. Foram realizadas visitas diárias à unidade de clínica cirúrgica para recrutamento dos participantes, por meio de convite verbal. O último participante foi recrutado em outubro de 2017.

Os dados foram coletados com auxílio de formulário de coleta de dados elaborado no Epi Info $^{\text {TM }}$ em dispositivo móvel (Tablet), com as seguintes variáveis: idade (em anos completos); horário da coleta (em horas, minutos e segundos); sexo (feminino e masculino); raça autodeclarada (branca e negra/parda); escore da Escala de Braden; temperatura do ambiente (em 
Temperatura da pele de diferentes áreas corporais de idosos sem risco para lesões por ... |6

$\left.{ }^{\circ} \mathrm{C}\right)$; umidade do ambiente (em \%); temperatura da pele (em ${ }^{\circ} \mathrm{C}$ ) de diferentes áreas corporais com proeminências ósseas - escápulas, cotovelos, trocanteres e calcâneos bilateralmente, occipital e sacral.

As áreas de proeminência óssea foram escolhidas, uma vez que são os locais mais propensos ao desenvolvimento de LP. São locais anatômicos onde as forças de fricção (perpendicular) e cisalhamento (tangencial), produzem maiores danos aos tecidos moles, tornando-os mais vulneráveis ao aparecimento de LP. ${ }^{19}$

A escolha por pacientes sem risco de desenvolver LP se deve ao fato de produzir parâmetros clínicos da temperatura normal para estas áreas corporais, pois é importante ressaltar que existem informações limitadas sobre a temperatura normal de pele sobre locais anatômicos propensos ao desenvolvimento de lesões de pressão. ${ }^{9}$ Selecionou-se o serviço de clínica cirúrgica, em virtude de ser a unidade hospitalar em que os pacientes permanecem internados e, principalmente, durante o pré-operatório, não possuem risco de desenvolvimento de LP. A escolha por pacientes idosos ocorreu pelo fato de a idade avançada ser um fator de risco para o desenvolvimento de LP. ${ }^{19-20}$

Para a mensuração da temperatura e umidade do ambiente, foi utilizado um termohigrômetro digital. Para a mensuração da temperatura da pele, o termógrafo infravermelho com laser de um só ponto, foi posicionado a sete centímetros de distância da pele ${ }^{6}$ nas áreas de proeminências ósseas já citadas; entre as 9:00 e 17:00h.

Os dados foram transferidos do Epi Info ${ }^{\mathrm{TM}}$ para planilha do Microsoft Excel e analisados com auxílio do Statistical Package for the Social Science (SPSS) for Windows, versão 21. Foi realizada análise estatística descritiva, empregando-se medidas de tendências centrais (média e mediana), de dispersão (desvio padrão e intervalo interquartil) e de proporção de acordo com a natureza das variáveis. Para análise da distribuição de normalidade utilizou-se teste de normalidade de Shapiro-Wilk e análise bivariada dos dados. Para verificar relação entre as variáveis independentes com a temperatura da pele, foi utilizado o teste t de Student (dados com 
7 | Soares RS, Lima SBS, Eberhardt TD, Silveira LBTD, Pozzebon BR, Rodrigues LR, et al.

distribuição normal, dois grupos independentes) ou U de Mann-Whitney (ausência de normalidade, dois grupos independentes); ANOVA de Friedman (ausência de normalidade, mais de dois grupos dependentes) e post hoc de Wilcoxon.

A correlação entre as variáveis quantitativas foi verificada por meio da correlação de Spearman. A interpretação da correlação foi realizada com base na Regra de Tumb, sendo considerada como uma correlação muito alta quando foram obtidos valores de $\mathrm{r}$ de 0,90 a 1,00; alta de 0,70 a 0,90 ; moderada de 0,50 a 0,70 ; baixa de 0,30 a 0,50 ; e insignificante de $0,00 .{ }^{18}$ Para efeito de análise, foi utilizado um nível de significância de $5 \%$.

Seguindo as normas e diretrizes regulamentadoras de pesquisa em seres humanos, o projeto de pesquisa foi aprovado pelo Comitê de Ética em Pesquisa sob parecer n⿳0 2.014.558 e Certificado de Apresentação para Apreciação Ética nº 66615417.9.0000.5346. Os participantes da pesquisa primária foram informados sobre o desenvolvimento da pesquisa e garantia de anonimato; aqueles que aceitaram participar assinaram o Termo de Consentimento Livre e Esclarecido em duas vias, sendo uma das vias entregues ao participante.

\section{Resultados}

Foram avaliados, para elegibilidade, 637 pacientes internados; após a aplicação dos critérios de seleção, foram incluídos na coleta 234. Para esta análise, foram excluídos quatro devido a dados faltantes na coleta de dados (missing data) e 146 devido à idade ( $<60$ anos), sendo incluídos 84 participantes - Figura 1. 
Temperatura da pele de diferentes áreas corporais de idosos sem risco para lesões por ... $\mid 8$

Figura 1 - Fluxograma de seleção dos participantes da pesquisa sobre temperatura da pele em idosos hospitalizados em unidade de clínica cirúrgica sem risco de desenvolver lesão por pressão, Santa Maria, RS, Brasil, 2017

Fluxograma de seleção dos participantes da pesquisa

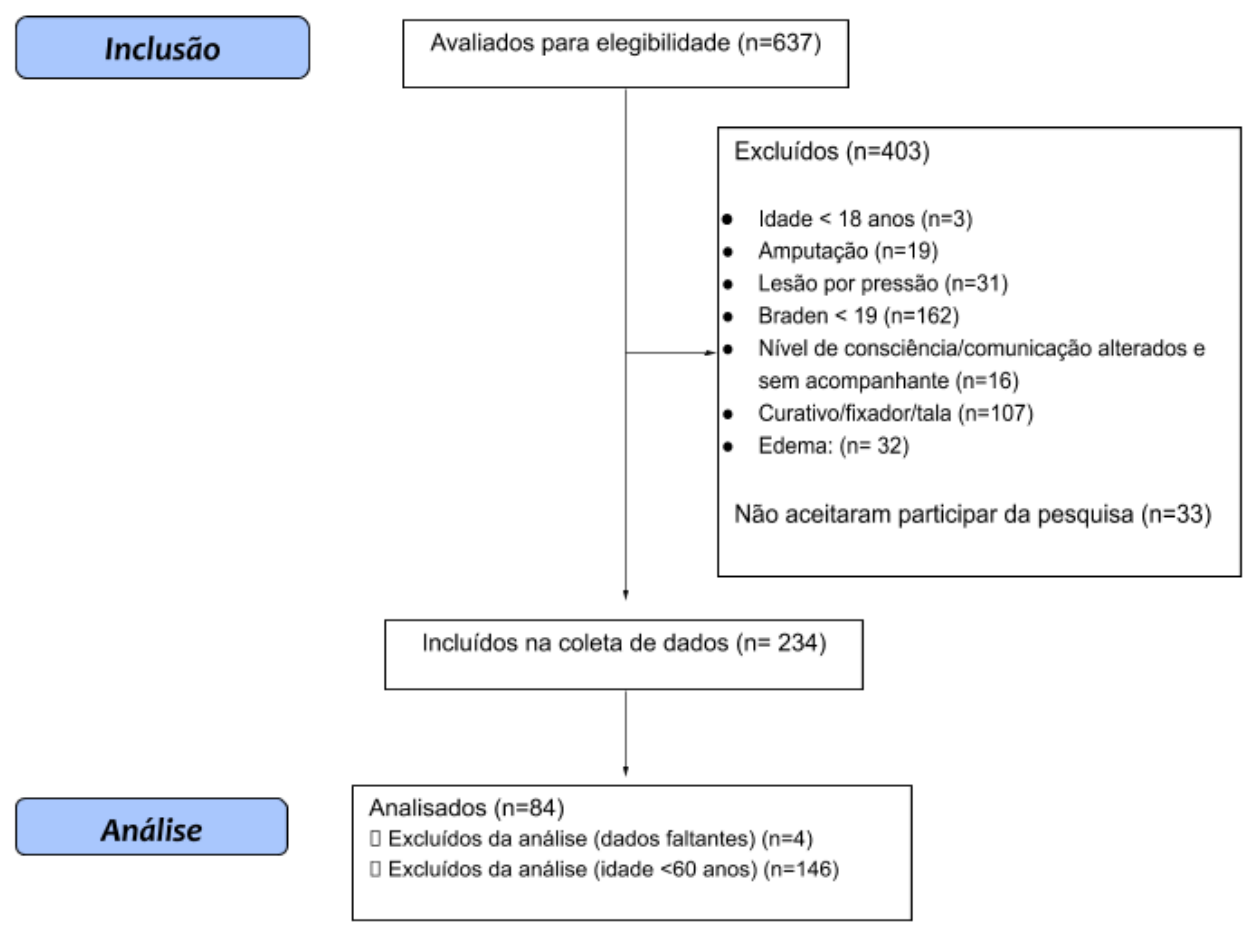

Foram avaliados 84 indivíduos, os quais se caracterizaram pela predominância do sexo masculino (65,5\%), raça branca $(79,8 \%)$ e idade compreendida entre 60 e 88 anos, com média de

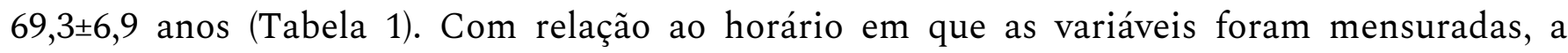
maioria $(76,2 \%)$ foi avaliada no horário compreendido das 13:01 às 17:00 horas. Na Tabela 1, também se observa o escore final da escala de Braden, apresentando uma pontuação que aponta para ausência de risco com relação ao desenvolvimento de LP $(22,2 \pm 1,2)$ pontos, com uma variação de 19 a 23. Com relação à temperatura e umidade do ambiente, verifica-se, respectivamente, uma média de $24,0^{\circ} \mathrm{C}$ e $64,0 \%$. 
9 | Soares RS, Lima SBS, Eberhardt TD, Silveira LBTD, Pozzebon BR, Rodrigues LR, et al.

Tabela 1 - Distribuição dos idosos hospitalizados em unidade de clínica cirúrgica sem risco de desenvolver lesão por pressão segundo as variáveis sexo, raça, horário das mensurações, idade, escore da Escala de Braden, temperatura e umidade do ambiente (n=84). Santa Maria, RS, Brasil, 2017.

\begin{tabular}{|c|c|c|}
\hline Variável & $\mathbf{n}$ & $(\%)$ \\
\hline \multicolumn{3}{|l|}{ Sexo } \\
\hline Masculino & 55 & 65,5 \\
\hline Feminino & 29 & 34,5 \\
\hline \multicolumn{3}{|l|}{ Raça } \\
\hline Branca & 67 & 79,8 \\
\hline Parda & 11 & 13,1 \\
\hline Negra & 6 & 7,1 \\
\hline \multicolumn{3}{|l|}{ Horário das mensurações } \\
\hline Das 9:00 às 13:00 horas & 20 & 23,8 \\
\hline \multirow[t]{2}{*}{ Das 13:01 às 17:00 horas } & 64 & 76,2 \\
\hline & \multicolumn{2}{|c|}{ Média $\pm D P^{*}\left(M^{\prime} n^{+}-M a ́ x *\right)$} \\
\hline Idade (em anos) & 69,3 & \\
\hline Escore da Escala de Braden & 22,2 & \\
\hline Temperatura do ambiente $\left({ }^{\circ} \mathrm{C}\right)$ & $24,0 \pm$ & \\
\hline Umidade do ambiente (\%) & $64,0 \pm$ & \\
\hline
\end{tabular}

Com relação à temperatura das áreas corporais de proeminências ósseas avaliadas (Tabela 2), observa-se que a região sacral foi a que apresentou média de temperatura mais alta $(34,5 \pm 1,8)$, com uma variação de $25,7^{\circ} \mathrm{C}$ a $38,0^{\circ} \mathrm{C}$. Enquanto a região do calcâneo direito apresentou a menor média $(28,1 \pm 2,9)$, com uma temperatura mínima de $22,5^{\circ} \mathrm{C}$ e máxima de $33,9^{\circ} \mathrm{C}$.

$\mathrm{Na}$ Tabela 2, pode-se observar que há diferença significativa $(\mathrm{p}<0,001)$ entre a temperatura da pele de todas as regiões corporais, exceto entre os lados direito e esquerdo nas regiões: escápulas, trocanteres e calcâneos. Essa ausência de diferença significativa entre os lados corporais indica simetria entre os lados direito e esquerdo; por esse motivo, as demais análises foram realizadas com a média da temperatura entre os lados das respectivas áreas corporais. Não foi encontrada diferença entre as temperaturas da pele dos trocanteres e região occipital. 
Temperatura da pele de diferentes áreas corporais de idosos sem risco para lesões por ... | 10

Tabela 2 - Comparação entre áreas corporais e temperatura da pele em idosos hospitalizados em unidade de clínica cirúrgica sem risco de desenvolver lesão por pressão (n=84). Santa Maria, RS, Brasil, 2017.

\begin{tabular}{|c|c|c|c|c|}
\hline \multirow{2}{*}{ Áreas corporais } & \multicolumn{2}{|c|}{ Temperatura da pele $\left({ }^{\circ} \mathrm{C}\right)$} & \multirow{2}{*}{$\begin{array}{c}\text { p- } \\
\text { valor" }\end{array}$} & \multirow{2}{*}{ Diferenças significativas"* } \\
\hline & $\begin{array}{l}\text { Média } \pm \mathrm{DP}^{*}\left(\text { Mín }^{+}-\right. \\
\left.\text {Máx }{ }^{*}\right)\end{array}$ & $\mathbf{M}^{\S}\left(\mathrm{IQ}^{\|}\right)$ & & \\
\hline Occipital & $32,4 \pm 1,6(27,9-35,9)$ & $32,5(1,9)$ & & $\begin{array}{l}\text { Occipital } \neq \text { todas as áreas; exceto trocanter } \\
\text { esquerdo e direito }\end{array}$ \\
\hline Escápula esquerda & $33,6 \pm 1,6(29,5-36,8)$ & $33,7(2,3)$ & & $\begin{array}{l}\text { Escápula esquerda } \neq \text { todas as áreas; exceto } \\
\text { escápula direita }\end{array}$ \\
\hline Escápula direita & $33,7 \pm 1,6(28,1-36,7)$ & $34,0(2,5)$ & & $\begin{array}{l}\text { Escápula direita } \neq \text { todas as áreas; exceto } \\
\text { escápula esquerda }\end{array}$ \\
\hline Cotovelo esquerdo & $31,4 \pm 1,6(23,4-35,8)$ & $31,6(2,2)$ & & Cotovelo esquerdo $\neq$ todas as áreas \\
\hline Cotovelo direito & $31,2 \pm 1,5(27,1-34,6)$ & $31,1(2,3)$ & & Cotovelo direito $\neq$ todas as áreas \\
\hline Sacral & $34,5 \pm 1,8(25,7-38,0)$ & $35,0(2,0)$ & $<0,001$ & Sacral $\neq$ todas as regiões \\
\hline Trocanter esquerdo & $32,8 \pm 1,5(28,8-35,9)$ & $33,0(1,9)$ & & $\begin{array}{l}\text { Trocanter esquerdo } \neq \text { todas as áreas; exceto } \\
\text { trocanter direito e occipital }\end{array}$ \\
\hline Trocanter direito & $32,9 \pm 1,6(27,5-35,9)$ & $33,0(2,3)$ & & $\begin{array}{l}\text { Trocanter direito } \neq \text { todas as áreas; exceto } \\
\text { trocanter esquerdo e occipital }\end{array}$ \\
\hline Calcâneo esquerdo & $28,4 \pm 2,7(20,5-33,8)$ & $28,3(3,6)$ & & $\begin{array}{l}\text { Calcâneo esquerdo } \neq \text { todas as áreas; exceto } \\
\text { calcâneo direito }\end{array}$ \\
\hline Calcâneo direito & $28,1 \pm 2,9(22,5-33,9)$ & $28,0(4,3)$ & & $\begin{array}{l}\text { Calcâneo direito } \neq \text { todas as áreas; exceto } \\
\text { calcâneo esquerdo }\end{array}$ \\
\hline
\end{tabular}

* DP=Desvio Padrão; ${ }^{+}$Mín=Valor mínimo; ${ }^{*}$ Máx=Valor máximo; § M=Mediana; || IQ=Intervalo Interquartil; ANOVA de Friedman, ${ }^{* *}$ Teste post hoc Wilcoxon, considerado um nível de significância de 5\%

Conforme descrito na Tabela 3, não há diferença significativa na temperatura da pele entre o sexo feminino e masculino, entre as raças autodeclaradas branca e parda/negra. Quanto ao horário de mensuração da temperatura da pele, a mensurada das 9:00 às 13:00h foi significativamente menor do que a mensurada das 13:01 às 17:00h na região dos calcâneos, p=0,005. 
11 | Soares RS, Lima SBS, Eberhardt TD, Silveira LBTD, Pozzebon BR, Rodrigues LR, et al.

Tabela 3 - Comparação entre sexo, raça, horário de mensuração e temperatura da pele em diferentes áreas corporais em idosos hospitalizados em unidade de clínica cirúrgica sem risco de desenvolver lesão por pressão (n=84). Santa Maria, RS, Brasil, 2017.

\begin{tabular}{|c|c|c|c|}
\hline \multirow{3}{*}{ Áreas corporais } & \multicolumn{2}{|c|}{ Temperatura da pele $\left({ }^{\circ} \mathrm{C}\right)$ por sexo } & \multirow[t]{3}{*}{ p-valor } \\
\hline & Feminino & Masculino & \\
\hline & Média $\pm \mathrm{DP}^{*}$ & Média $\pm D P$ & \\
\hline Occipital & $31,9 \pm 2,1$ & $32,6 \pm 1,2$ & $0,087^{\ddagger}$ \\
\hline Escápulas & $33,4 \pm 1,6$ & $33,8 \pm 1,5$ & $0,268^{\neq}$ \\
\hline Cotovelos & $31,4 \pm 1,3$ & $31,2 \pm 1,5$ & $0,378^{p^{*}}$ \\
\hline Sacral & $33,9 \pm 2,4$ & $34,8 \pm 1,3$ & $0,071^{\dagger}$ \\
\hline Trocanteres & $32,6 \pm 1,2$ & $33,0 \pm 1,5$ & $0,233^{\ddagger}$ \\
\hline \multirow[t]{2}{*}{ Calcâneos } & $27,9 \pm 4,9$ & $28,5 \pm 2,8$ & $0,352^{\ddagger}$ \\
\hline & \multicolumn{2}{|c|}{ Temperatura da pele $\left({ }^{\circ} \mathrm{C}\right)$ por raça } & p-valor \\
\hline \multirow[t]{2}{*}{ Áreas corporais } & Branca & Parda/negra & \\
\hline & Média $\pm \mathrm{DP}$ & Média $\pm D P$ & \\
\hline Occipital & $32,4 \pm 1,6$ & $32,4 \pm 1,6$ & $0,639^{+}$ \\
\hline Escápulas & $33,7 \pm 1,6$ & $33,4 \pm 1,2$ & $0,449^{\ddagger}$ \\
\hline Cotovelos & $31,3 \pm 1,5$ & $31,2 \pm 1,3$ & $0,987^{*}$ \\
\hline Sacral & $34,6 \pm 1,6$ & $34,3 \pm 2,4$ & $0,941^{+}$ \\
\hline Trocanteres & $32,8 \pm 1,5$ & $33,0 \pm 0,9$ & $0,703^{\ddagger}$ \\
\hline Calcâneos & $28,4 \pm 2,5$ & $27,6 \pm 3,1$ & $0,228^{\ddagger}$ \\
\hline \multirow{3}{*}{ Áreas corporais } & \multicolumn{2}{|c|}{ Temperatura da pele $\left({ }^{\circ} \mathrm{C}\right)$ mensurada em diferentes horários } & p-valor \\
\hline & 9:00-13:00h & 13:01-17:00h & \\
\hline & Média $\pm \mathrm{DP}$ & Média $\pm D P$ & \\
\hline Occipital & $32,0 \pm 1,6$ & $32,5 \pm 1,6$ & $0,682^{+}$ \\
\hline Escápulas & $33,6 \pm 1,7$ & $33,7 \pm 1,5$ & $0,679^{\ddagger}$ \\
\hline Cotovelos & $30,9 \pm 1,3$ & $31,4 \pm 1,4$ & $0,157^{\ddagger}$ \\
\hline Sacral & $34,3 \pm 1,8$ & $34,6 \pm 1,8$ & $0,194^{+}$ \\
\hline Trocanteres & $32,3 \pm 1,3$ & $33,0 \pm 1,4$ & $0,089^{*}$ \\
\hline Calcâneos & $27,0 \pm 1,9$ & $28,7 \pm 2,7$ & $0,004^{\ddagger}$ \\
\hline
\end{tabular}

${ }^{*} \mathrm{DP}=$ Desvio padrão; ${ }^{+}$Teste U de Mann-Whitney, considerado um nível de significância de $5 \%$.

${ }^{*}$ Teste $\mathrm{T}$ de Student, considerado um nível de significância de 5\%.

A Tabela 4 apresenta dados de correlação entre idade, temperatura do ambiente, umidade do ambiente e temperatura da pele em diferentes áreas corporais dos idosos. 
Temperatura da pele de diferentes áreas corporais de idosos sem risco para lesões por ... | 12

Tabela 4 - Correlação entre idade, temperatura do ambiente, umidade do ambiente e temperatura da pele em diferentes áreas corporais em idosos hospitalizados em unidade de clínica cirúrgica sem risco de desenvolver lesão por pressão (n=84). Santa Maria, RS, Brasil, 2017.

\begin{tabular}{lcccccc}
\hline \multirow{2}{*}{$\begin{array}{l}\text { Área de mensuração da } \\
\text { temperatura da pele }\end{array}$} & \multicolumn{2}{c}{ Idade } & \multicolumn{2}{c}{$\begin{array}{c}\text { Temperatura do } \\
\text { ambiente }\end{array}$} & \multicolumn{2}{c}{$\begin{array}{c}\text { Umidade do } \\
\text { ambiente }\end{array}$} \\
\cline { 2 - 8 } & $\boldsymbol{\rho}^{\boldsymbol{*}}$ & $\mathbf{p}$-valor & $\boldsymbol{\rho}$ & $\mathbf{p}$-valor & $\boldsymbol{\rho}$ & p-valor \\
\hline Occipital & 0,011 & 0,921 & $0,355^{+}$ & 0,001 & $-0,078$ & 0,482 \\
Escápulas & $-0,133$ & 0,227 & $-0,063$ & 0,571 & 0,088 & 0,426 \\
Cotovelos & 0,071 & 0,523 & 0,207 & 0,059 & $-0,160$ & 0,145 \\
Sacral & $-0,061$ & 0,579 & 0,069 & 0,534 & 0,014 & 0,899 \\
Trocanteres & $-0,065$ & 0,555 & $-0,069$ & 0,535 & 0,047 & 0,674 \\
Calcâneos & 0,020 & 0,857 & $0,220^{+}$ & 0,044 & $-0,237^{+}$ & 0,030 \\
\hline
\end{tabular}

* $\rho$ : Coeficiente de Correlação de Spearman.

${ }^{+}$Correlações significativas, a nível de significância de 5\%.

Foi encontrada correlação positiva baixa entre a temperatura da pele da região occipital e a temperatura do ambiente $(\rho=0,355 ; p=0,001)$. As correlações entre a temperatura dos calcâneos e a temperatura do ambiente, e entre a temperatura dos calcâneos e a umidade do ambiente, são consideradas insignificantes $(\rho<0,300 ; p<0,05)$ - Tabela 4 .

\section{Discussão}

Dados epidemiológicos indicam que as LPs ocorrem em todas as faixas etárias, mas estão associadas ao aumento da idade. ${ }^{21}$ A pele é um órgão constituído de camadas: epiderme e derme. O estrato córneo é a camada mais externa da epiderme, portanto é a primeira camada diretamente afetada por qualquer alteração de temperatura ou umidade. ${ }^{9}$ A temperatura da pele é uma medida fisiológica que pode servir como parâmetro clínico na prevenção de LP, uma vez que um aumento em $1,2^{\circ} \mathrm{C}$ em 24 a 96 horas pode acontecer antes do aparecimento de LP. ${ }^{22}$

O avanço da idade promove diversas e profundas transformações na pele. Destaca-se no idoso uma maior fragilidade cutânea e menor capacidade de atuar como barreira contra fatores 
13 | Soares RS, Lima SBS, Eberhardt TD, Silveira LBTD, Pozzebon BR, Rodrigues LR, et al.

externos. A pele apresenta-se mais seca e rugosa devido à redução do número de glândulas sebáceas, diminuição da elasticidade, flacidez, alteração da resposta imunológica celular e diminuição da espessura da derme e da epiderme, aumentando o risco de lesões. ${ }^{23}$

Estudo realizado com o objetivo de determinar a distribuição de calor nos membros superiores e inferiores em uma população de adultos saudáveis evidencia que existe uma simetria geral, em termos de temperaturas médias para ambos os lados do corpo dos participantes. ${ }^{24}$ Logo, corrobora os achados desta pesquisa, uma vez que esta simetria entre os lados corporais também foi identificada.

Destaca-se que, mesmo havendo simetria nos valores das temperaturas entre os lados corporais, observa-se que há diferença significativa $(\mathrm{p}<0,001)$ entre a temperatura da pele de todas as regiões corporais. No entanto, destacam-se achados de outra pesquisa a qual apresenta que as temperaturas médias de ambos os lados dos participantes foram similares nas extremidades, tais como dedos dos pés e das mãos e as superfícies palmares e plantares. $(P<0,05) .{ }^{24}$

As médias da temperatura e umidade do ambiente, nesta pesquisa, foram de $24,0^{\circ}$ e $64 \%$ respectivamente; essas condições foram semelhantes a de outros estudos realizados com pacientes internados em Unidade de Tratamento Intensivo ${ }^{6}$ e com indivíduos hígidos trabalhadores da Força Aérea Brasileira. Com relação à temperatura das diferentes áreas corporais, destaca-se a temperatura da pele da região sacral $\left(34,5^{\circ} \mathrm{C}\right)$ e calcâneos direito $\left(28,1^{\circ} \mathrm{C}\right)$ e esquerdo $\left(28,4^{\circ} \mathrm{C}\right)$, por serem consideradas locais de maior incidência de LP..$^{25}$

A região dos calcâneos é mais propensa ao desenvolvimento das lesões. A área da superfície de contato no calcanhar é pequena e apresenta pouco tecido subcutâneo, sendo a pressão exercida diretamente no osso calcâneo. Associadas a essa característica, as propriedades vasculares fazem com que a pressão aplicada sobre a região cause um sério comprometimento à perfusão. ${ }^{26}$

Estudo desenvolvido com o objetivo de comparar a temperatura da pele de idosas e jovens, fisicamente ativas, usando termografia, entre os lados do corpo, evidencia que mulheres 
Temperatura da pele de diferentes áreas corporais de idosos sem risco para lesões por ... | 14

mais velhas apresentam temperatura da pele mais alta que as jovens, e as maiores diferenças estão concentradas em regiões periféricas do corpo, como as mãos. Independentemente da faixa etária, existe uma simetria térmica bilateral geralmente inferior a $0,5^{\circ} \mathrm{C}$ em mulheres jovens e idosas. No entanto, mulheres idosas apresentam valores mais altos de temperatura da pele que as mulheres jovens na maioria das regiões avaliadas, principalmente nas regiões distais dos membros. ${ }^{27}$

Neste estudo, não existe diferença significativa na temperatura da pele entre o sexo feminino e masculino. Com estudantes universitários, realizou-se uma pesquisa em sete locais do corpo (braço, tórax, costas, barriga, cintura, coxa e perna), a qual investigou a temperatura da pele e o fluxo de calor em ambiente de laboratório. Com o aumento da temperatura do ambiente de 25 a $31^{\circ} \mathrm{C}$, as temperaturas locais da pele também aumentaram gradualmente. A temperatura da pele da cintura e barriga para o grupo feminino foram maiores do que para o grupo masculino. As temperaturas da pele das pernas, coxas e costas para ambos os grupos se mostraram semelhantes. $^{28}$

Com relação ao horário de mensuração da temperatura da pele, identificou-se que a mensurada das 9:00 às 13:00h foi significativamente menor do que a mensurada das 13:01 às 17:00h na região dos calcâneos. Este achado vai ao encontro dos resultados de outro estudo, que sinaliza um comportamento diferente da temperatura em áreas distintas do corpo, com variações entre o final da tarde e a noite, constatando temperaturas mais altas às 23:00h. ${ }^{29}$ Este fato pode estar associado à influência do ritmo circadiano, que é definido como as mudanças fisiológicas, metabólicas e comportamentais que ocorrem no corpo humano no período de 24 horas. É controlado pelo núcleo supraquiasmático (SCN) do hipotálamo anterior e é grandemente influenciado pela luz e pelo meio ambiente. ${ }^{30}$

O ritmo circadiano controla nossa temperatura corporal e temperatura da pele. A temperatura corporal central é mais alta durante o dia, quando comparada ao horário noturno, 
15 | Soares RS, Lima SBS, Eberhardt TD, Silveira LBTD, Pozzebon BR, Rodrigues LR, et al.

com uma diminuição no início da manhã; já a pele apresenta aumento de temperatura à tarde com uma diminuição à noite, o que corrobora com os achados deste estudo. ${ }^{30}$

A avaliação da temperatura da pele é uma estratégia que pode auxiliar os profissionais de saúde a detectar atividade metabólica alterada. Um aumento nessa variável resulta em um aumento de $10 \%$ no metabolismo celular, o que por sua vez, leva a danos nos tecidos já que reduz o fluxo sanguíneo. ${ }^{22}$ Logo, sua mensuração auxilia na predição de risco para lesão por pressão. ${ }^{3-8,22}$

Assim, no contexto da prevenção das LPs, os achados deste estudo são importantes para fortalecer valores de referência da temperatura para cada região do corpo, uma vez que, como se sabe, podem ocorrer alterações da temperatura da pele anteriores ao aparecimento de manifestações clínicas na pele relacionadas as LP. ${ }^{7-8}$

Sugere-se a realização de estudos que avaliem a correlação entre idade e temperatura da pele, além de estudar a influência da temperatura e umidade do ambiente na temperatura da pele de diferentes áreas corporais. Este estudo possui, como limitação, a amostragem não probabilística, podendo configurar um viés de seleção dos participantes da pesquisa.

\section{Conclusão}

Conclui-se que, por meio das medidas da temperatura da pele, foi possível verificar os seguintes parâmetros das diferentes áreas corporais: a região sacral foi a que apresentou média de temperatura mais alta $(34,5 \pm 1,8)$, enquanto a região do calcâneo direito apresentou a menor média $(28,1 \pm 2,9)$. Todas as regiões mensuradas apresentam diferenças nas temperaturas, exceto os lados direito e esquerdo nas escápulas, trocanteres e calcâneo, indicando simetria entre os lados do corpo e entre a região occipital e os trocanteres. Quanto aos horários de mensuração, destaca-se que as mensurações realizadas à tarde (13h01min às 17:00h) apresentaram temperaturas maiores na região dos calcâneos. 
Temperatura da pele de diferentes áreas corporais de idosos sem risco para lesões por ... | 16

Os resultados encontrados contribuem para o avanço do conhecimento acerca da temperatura da pele de idosos, com importantes implicações para a gestão do cuidado relacionado à prevenção de lesão por pressão nessa população. O objetivo é o conhecimento produzido facilite a tomada de decisão clínica do profissional no seu fazer cotidiano, uma vez que há evidências de que alterações na temperatura da pele sugerem o aparecimento de LP.

Assim, este estudo apresenta dados de diferentes áreas de proeminência óssea de população idosa, que são as áreas e indivíduos de maior risco de desenvolvimento de LP. Dessa forma, os dados evidenciados poderão servir como parâmetros clínicos para futuras metaanálises sobre a temática.

\section{Referências}

1. Mescher AJ. Basic Histology: text and atlas. 14 $4^{\text {th }}$ ed. Texas: McGraw-Hill Education; 2016.

2. Souza NR, Freire DA, Souza MAO, Santos ICRV, Santos LV, Bushatsky M. Fatores predisponentes para o desenvolvimento da lesão por pressão em pacientes idosos: uma revisão integrativa. Estima. 2017;15(4):229-39. doi: https://doi.org/10.5327/Z1806-3144201700040007

3. Gefen A. How do microclimate factors affect the risk for superficial pressure ulcers: a mathematical modeling study. J Tissue Viability. 2011;20:81-8. doi: https://doi.org/10.1016/j.jtv.2010.10.002

4. Kottner J, Dobos G, Andruck A, Trojahn C, Apelt J, Wehrmeyer H, et al. Skin response to sustained loading: a clinical explorative study. J Tissue Viability. 2015;24(3):114-22. doi: https://doi.org/10.1016/j.jtv.2015.04.002

5. Lachenbruch C, Tzen YT, Brienza D, Karg PE, Lachenbruch PA. Relative contributions of interface pressure, shear stress, and temperature on ischemic-induced, skin-reactive hyperemia in healthy volunteers: a repeated measures laboratory study. Ostomy Wound Manage. 2015;61(2):16-25.

6. Yusuf S, Okuwa M, Shigeta Y, Dai M, Iuchi T, Rahman S, et al. Microclimate and development of pressure ulcers and superficial skin changes. Int Wound J. 2015;12(1):40-6. doi: https://doi.org/10.1111/iwj.12048

7. Cox J, Kaes L, Martinez M, Moles D. A prospective, observational study to assess the use of thermography to predict progression of discolored intact skin to necrosis among patients in skilled nursing facilities. Ostomy Wound Manage [Internet]. 2016 [cited 2020 Oct 20];62(10):14-33. Available 
17 | Soares RS, Lima SBS, Eberhardt TD, Silveira LBTD, Pozzebon BR, Rodrigues LR, et al.

from: https://www.o-wm.com/article/prospective-observational-study-assess-use-thermography-predictprogression-discolored

8. Agrawal M, Pardasani KR. Finite element model to study temperature distribution in skin and deep tissues of human limbs. J Therm Biol. 2016;62:98-105. doi: https://doi.org/10.1016/j.jtherbio.2016.07.006

9. Kottner J, Black J, Call E, Gefen A, Santamaria N. Microclimate: a critical review in the context of pressure ulcer prevention. Clin Biomech (Bristol Avon). 2018;59:62-70. doi: https://doi.org/10.1016/j.clinbiomech.2018.09.010

10. WoundSource. National Pressure Ulcer Advisory Panel (NPUAP) announces a change in terminology from pressure ulcer to pressure injury and updates the stages of pressure injury [Internet]. National Pressure Ulcer Advisory Panel: Washington; 2016 [cited 2019 Oct 19]. Available from: https://www.woundsource.com/blog/national-pressure-ulcer-advisory-panel-npuap-announces-changein-terminology-pressure-ulcer

11. Soares CF, Heidemann ITSB. Promoção da saúde e prevenção da lesão por pressão: expectativas do enfermeiro da atenção primária. Texto Contexto Enferm. 2018;27(2). doi: https://doi.org/10.1590/0104070720180001630016

12. Jesus MAP, Pires PS, Biondo CS, Matos RM. Incidência de lesão por pressão em pacientes internados e fatores de risco associados. Rev Baiana Enferm. 2020;34:e36587. doi: https://doi.org/10.18471/rbe.v34.36587

13. Constantin AG, Moreira APP, Oliveira JLC, Hofstätter LM, Fernandes LM. Incidência de lesão por pressão em unidade de terapia intensiva para adultos. Estima. 2017;16:e1118. doi: https://doi.org/10.30886/estima.v16.454_PT

14. European Pressure Ulcer Advisory Panel; National Pressure Injury Panel; Pan Pacific Pressure Injury Alliance. Prevention and treatment of pressure ulcers/injuries: quick reference guide. Emily Haesler: EPUAP/NPIAP/PPPIA; 2019.

15. Paranhos WY, Santos VLCG. Avaliação de risco para úlceras de pressão por meio da escala de Braden, na língua portuguesa. III Congresso Brasileiro de Estomaterapia; 1999 nov 09-13 [acesso em 20 out 2020]. São Paulo, SP. (Rev Esc Enferm USP [Internet];33(N Esp):191-206). Disponível em: http://www.ee.usp.br/reeusp/upload/pdf/799.pdf

16. Wong V. Heel response to external pressure is it the same in healthy community-dwelling adults and in hip surgery patients? J Wound Ostomy Continence Nurs. 2014;41(6):539-48. doi: https://doi.org/10.1097/WON.0000000000000067

17. Polit DF, Beck CT. Fundamentos de pesquisa em enfermagem: avaliação de evidências para a prática de enfermagem. $7^{\mathfrak{a}}$ ed. Porto Alegre: Artmed; 2011. 
Temperatura da pele de diferentes áreas corporais de idosos sem risco para lesões por ... | 18

18. BRASIL. Ministério da Saúde. Estatuto do Idoso. $3^{\underline{a}}$ ed. Brasília, DF: Ministério da Saúde, 2013. 72 p. Disponível em: http://bvsms.saude.gov.br/bvs/publicacoes/estatuto_idoso_3edicao.pdf. Acesso em 20 out. 2020.

19. Coleman S, Nixon J, Keen J, Wilson L, McGinnis E, Dealey C, et al. A new pressure ulcer conceptual framework. J Adv Nurs. 2014;70(10):2222-34. doi: https://doi.org/10.1111/jan.12405

20. Scott SM. Progress and challenges in perioperative pressure ulcer prevention. J Wound Ostomy Continence Nurs. 2015;42(5):480-5. doi: https://doi.org/10.1097/WON.0000000000000161

21. Hahnel E, Lichterfeld A, Blume-Peytavi U, Kottner J. The epidemiology of skin conditions in the aged: a systematic review. J Tissue Viability. 2017;26(1):20-8. doi: https://doi.org/10.1016/j.jtv.2016.04.001

22. Sae-Sia W, Wipke-Tevis DD, Williams DA. Elevated sacral skin temperature (Ts): A risk factor for pressure ulcer development in hospitalized neurologically impaired Thai patients. Appl Nurs Res. 2005;18(1):29-35. doi: https://doi.org/10.1016/j.apnr.2004.03.005

23. Domansky RC, Borges EL. Manual para prevenção de lesões de pele: recomendações baseadas em evidências. $2^{\underline{a}}$ ed. Rio de Janeiro: Rubio; 2014.

24. Gatt A, Formosa C, Cassar K, Camilleri KP, De Raffaele C, Mizzi A, et. Al. Thermographic patterns of the upper and lower limbs: baseline data. Int J Vasc Med. 2015;831369. doi: https://doi.org/10.1155/2015/831369

25. Engels D, Austin M, McNichol L, Fencl J, Gupta S, Kazi H. Pressure ulcers: factors contributing to their development in the OR. AORN J. 2016;103(3):271-81. doi: https://doi.org/10.1016/j.aorn.2016.01.008

26. Gefen A. Why is the heel particularly vulnerable to pressure ulcers? Br J Nurs. 2017;26(Suppl 20):6274. doi: https://doi.org/10.12968/bjon.2017.26.Sup20.S62

27. Moreira DG, Molinari AB, Fernandes AA, Sillero-Quintana M, Brito CJ, Doimo LA, et al. Skin temperature of physically active elderly and young women measured using infrared thermography. J Physic Educ Sport. 2017;17(4):2531-7. doi: https://doi.org/10.7752/jpes.2017.04286

28. Wang L, Tian Y, Kim J, Yin H. The key local segments of human body for personalized heating and cooling. J Therm Biol. 2017;81:118-27. doi: https://doi.org/10.1016/j.jtherbio.2019.02.013

29. Costa CMA, Sillero-Quintana M, Piñonosa CS, Moreira DG, Brito CJ, Fernandes AA, et al. Daily oscillations of skin temperature in military personnel using thermography. BMJ Mil Health. 2016;162(5):335-42. doi: https://doi.org/10.1136/jramc-2015-000462

30. Lyons AB, Moy L, Moy R, Tung R. Circadian rhythm and the skin: a review of the literature. J Clin Aesthet Dermatol [Internet]. 2019 [cited 2020 Oct 20];12(9):42-5. Available from: https://www.ncbi.nlm.nih.gov/pmc/articles/PMC6777699/ 
19 | Soares RS, Lima SBS, Eberhardt TD, Silveira LBTD, Pozzebon BR, Rodrigues LR, et al.

Editor Científico: Tânia Solange Bosi de Souza Magnago

Editor Associado: Graziele de Lima Dalmolin

\section{Autor correspondente}

Rhea Silvia de Avila Soares

E-mail: rheasilviasoares@yahoo.com.br

Endereço: Jorge Pedro Abelin 432

CEP:97050-390

\section{Contribuições de Autoria}

\section{1 - Rhea Silvia Soares}

Concepção ou desenho do estudo/pesquisa, análise e/ou interpretação dos dados, revisão final com participação crítica e intelectual no manuscrito.

\section{2 - Suzinara Beatriz Soares de Lima}

Concepção ou desenho do estudo/pesquisa, análise e/ou interpretação dos dados, revisão final com participação crítica e intelectual no manuscrito.

\section{3 - Thaís Dresch Eberhardt}

Concepção ou desenho do estudo/pesquisa, análise e/ou interpretação dos dados, revisão final com participação crítica e intelectual no manuscrito.

\section{4 - Lidiana Batista Teixeira Dutra Silveira}

Concepção ou desenho do estudo/pesquisa, análise e/ou interpretação dos dados, revisão final com participação crítica e intelectual no manuscrito.

\section{5 - Bruna Rossarola Pozzebon}

Concepção ou desenho do estudo/pesquisa, análise e/ou interpretação dos dados, revisão final com participação crítica e intelectual no manuscrito.

\section{6 - Liane Rocha Rodrigues}

Concepção ou desenho do estudo/pesquisa, análise e/ou interpretação dos dados, revisão final com participação crítica e intelectual no manuscrito.

\section{7 - Paulo Jorge Pereira Alves}

Concepção ou desenho do estudo/pesquisa, análise e/ou interpretação dos dados, revisão final com participação crítica e intelectual no manuscrito.

\section{Como citar este artigo}

Soares RS, Lima SBS, Eberhardt TD, Silveira LBTD, Pozzebon BR, Rodrigues LR, et al. Temperatura da pele de diferentes áreas corporais de idosos sem risco para lesões por pressão. Rev. Enferm. UFSM. 2020 [Acesso em: Anos Mês Dia]; vol.10 e98: 1-19. DOI:https://doi.org/10.5902/2179769241643 